\title{
Propuesta Metodológica para Proyectos de Infraestructura Tecnológica en Trabajos de Titulación
}

\author{
Jessica J. Morales, Luis C. Cedeño, Jorge A. Parraga-Alava y Bethsy A. Molina \\ Escuela Superior Politécnica Agropecuaria de Manabí Manuel Félix López, Campus Politécnico, Sitio el \\ Limón, Calceta Manabí, Ecuador (e-mail: jessjohannamor@hotmail.com; Icedeno@espam.edu.ec; \\ jparraga@outlook.cl; alitaquinex1202@hotmail.com)
}

\section{Resumen}

Recibido Oct. 16, 2017; Aceptado Dic. 20, 2017; Versión final Feb. 7, 2018, Publicado Ago. 2018

\begin{abstract}
La investigación que se presenta tuvo como objetivo diseñar una metodología en soluciones de infraestructura aplicable a proyectos de implementación tecnológica. Para el desarrollo, se empleó el método cadena documental, que consta de tres etapas: Colecta, tratamiento y difusión. En la colecta, se recopiló información relevante de los procesos metodológicos empleados en trabajos de titulación desarrollados en la Carrera de Computación de la Escuela Superior Politécnica Agropecuaria de Manabí Manuel Félix López hasta el año 2015. En la etapa de tratamiento, se realizó un estudio descriptivo de casos de las metodologías empleadas en la sublínea de investigación de infraestructura tecnológica. En la difusión, se socializó la propuesta diseñada. La investigación muestra que los procesos metodológicos empleados para la ejecución de proyectos de infraestructura tecnológica fueron aceptables en apenas un $20 \%$. Esto demuestra que la metodología propuesta plantea claridad y precisión.
\end{abstract}

Palabras clave: desarrollo de proyectos; tecnologías de información; metodología tecnológica; infraestructura tecnológica; cadena documental

\section{Methodological Proposal for Technological Infrastructure Projects in Degree Thesis}

\begin{abstract}
The paper describes the design of a methodology in infrastructure solutions applicable to technological implementation projects. For the development, the documentary chain method was used, which consists of three stages: collection, treatment and diffusion. In the collection step relevant information is collected from the methodological processes used in degree thesis developed in the Computing Major of the Escuela Superior Politécnica Agropecuaria de Manabí Manuel Félix López, until 2015. In the treatment step, a descriptive case study of the methodologies used in the technological infrastructure research subline was carried out. In the diffusion step, the proposal was socialized. The research shows that the methodological processes used for the execution of technological infrastructure projects are acceptable in only $20 \%$. This demonstrates that the proposed methodology presents clarity and precision.
\end{abstract}

Keywords: development of projects; information technologies; technological methodology; technological infrastructure; documentary chain 


\section{INTRODUCCIÓN}

La investigación tecnológica, adquiere un espacio que trasciende de manera ineludible en las sociedades del mundo entero, y cada vez es mayor el consenso de que la humanidad vive desde finales del siglo pasado una revolución digital (Torres-Velandia et al., 2017). La tecnología es un recurso que sirve como base fundamental para el comportamiento productivo dentro de los sistemas económicos y sociales (Mair, 2016), por tanto, es considerado un factor que influye hacia el fortalecimiento de toda empresa, ocupando un papel fundamental para la competitividad entre las organizaciones (Pereira y Oliveira, 2016). El proceso de innovación tecnológica comprende un complejo conjunto de actividades que trasforman las ideas y el conocimiento científico en realidad física y aplicaciones del mundo real (Agostinho, 2015) esto requiere de una gran inversión, en tiempo de ejecución y ciclos de vida (Montesinos-Valera et al., 2017) por ello, se considera primordial, que exista una conexión entre las empresas y las instituciones que trabajan con innovación (Da Silva et al., 2016).

La empresa, el estado y la universidad, deben crear condiciones regionales especiales y políticas públicas (Scheel y Bretones, 2011) que garanticen el compromiso entre estos tres pilares de la sociedad, siendo de este modo, la academia el principal contribuyente a la generación de innovaciones, mediante la investigación y aplicación de tecnologías. En consecuencia, la empresa (sea pública o privada) está precisada a detectar cambios en el entorno, reaccionar en consecuencia a estos cambios, adaptarse e incluso, predecir los cambios (Navia et al., 2018) así como la gestión adecuada de la información donde se debe incluir la preocupación por su preservación y futura reutilización (Porcal-Gonzalo, 2015). Las empresas innovadoras buscan ideas de cualquier tipo y proporcionan una cultura organizacional que respalda su desarrollo (Agostinho, 2015), ideas que responden a las necesidades de la sociedad y el mercado (Carrillo et al., 2017).

Actualmente, el Estado ecuatoriano fomenta el desarrollo de territorios innovadores y ciudades creativas e inteligentes, generando redes de conocimiento y vinculando la educación superior con las necesidades sociales y productivas; e impulsa programas de investigación, formación, capacitación y actualización que respondan a las potencialidades y necesidades territoriales (Plan Nacional de Desarrollo, 2017), y son las universidades a través de las propuestas de proyectos de investigación o vinculación, de grado o postgrado, que deben estar enmarcados directamente con las necesidades de las comunidades sociales, las empresas y sus entornos locales articulando un desarrollo armónico y equilibrado (Scheel y Bretones, 2011).

De este modo, cada vez surgen más desarrollos de proyectos tecnológicos, que emergen de la necesidad de mejorar las ciencias aplicadas, donde la complejidad y el dimensionamiento de la información a procesar, hace necesario el uso de herramientas tecnológicas, que simplifiquen acciones y mejoren la calidad de vida del ser humano. Estos proyectos desarrollados desde la académica exigen como primer paso hacia la calidad del producto, el proceso de desarrollo (Plinio et al., 2014), por tanto, demanda el uso de metodologías aplicadas a dicho contexto, que garanticen la culminación exitosa del trabajo propuesto. Una metodología exige valorar los procesos que se generan, y en muchos casos suelen ser demasiados complejos para que se puedan capturar todas sus características, potencialmente importantes (Petty et al., 2012).

Los desafíos de una investigación, exigen que tanto los métodos como la metodología sean entendidos y asumidos como herramientas útiles que permiten el abordaje, la comprensión y la argumentación de los problemas de estudio (Aguilera, 2013), situación que puede tornarse compleja al estar frente a múltiples aplicaciones (Martelo et al., 2015) que se suscitan en proyectos de desarrollo tecnológico, debiendo ser aplicables y compatibles con proyectos orientados a tecnologías de telecomunicaciones, redes celulares, comunicación inalámbrica óptica, comunicaciones por satélite, internet y sistemas críticos de infraestructura informática y de almacenamiento; es importante contar con guías de trabajo y procesos para gestionar un campo tan vasto (Ley-Bosch et al., 2016 y De Oliveira et al., 2015).

La tecnología, dentro de estas múltiples áreas ofrece ventajas destacando: agilizar procesos, ahorrar costos, ahorrar personal, promover la educación. Dichas tecnologías son desarrolladas por expertos, basándose en métodos, modelos, diagramas y requerimientos (Martelo et al., 2017). Es por ello que al proponer investigaciones en proyectos tecnológicos hay que tener en cuenta la metodología que se implementará ya que de esto depende el éxito o fracaso del proyecto. Las metodologías, no siempre llenan las expectativas de los investigadores, debido a la falta de claridad en los procesos de desarrollo (Plinio et al., 2014), por tal razón se han ido creando nuevas metodologías que cumplan con los requisitos funcionales de estos procesos (Martelo et al., 2015) y que satisfagan las necesidades que aún no han sido cubiertas, esto ocurre permanentemente en centros de investigaciones, empresas y universidades.

La Escuela Superior Politécnica Agropecuaria de Manabí Manuel Félix López (ESPAM MFL) es una Institución de Educación Superior (IES) que todos los años aporta a la comunidad con proyectos en las áreas de Ingeniería Agrícola, Ingeniera Ambiental, Agroindustrias, Administración de empresas, Administración 
Pública, Medicina Veterinaria, Turismo y Computación. La Carrera de Computación ha emprendido esfuerzos generalizados para aumentar el número de estudiantes formados y garantizar contribuciones académicas a la sociedad que se enmarquen en esta área. A pesar de ello, se aprecia inconsistencia en los procesos metodológicos aplicados en los proyectos propuestos por los alumnos, que al momento de seleccionar el o los métodos para sus investigaciones (proyectos de año, trabajos de titulación, informes de pasantías) no se efectúa de manera pertinente, esto se percibe con frecuencia en aquellas orientadas a infraestructura tecnológica, haciendo uso inapropiado del sustento teórico, adaptan su necesidad de emplear una metodología hacia la que más se asemeje a su propuesta, siendo en la mayoría de los casos una metodología convencional o ágil orientada exclusivamente al desarrollo de software.

Teniendo en cuenta los antecedentes planteados, esta investigación consiste en el diseño de una metodología aplicable a proyectos de infraestructura tecnológica, sencilla, fácil de comprender y aplicar, y que al mismo tiempo cumpla con todos los ámbitos que requieren los proyectos tecnológicos en su ejecución; a esta propuesta metodológica se la denominó Eder.

\section{METODOLOGÍA}

Para realizar la propuesta del diseño de la metodología Eder, se inició desarrollando una investigación donde se fundamenta la problemática planteada en la Carrera de Computación de la Escuela Superior Politécnica Agropecuaria de Manabí Manuel Félix López ubicada en el cantón Bolívar, provincia Manabí - Ecuador, para ello se empleó el método Cadena Documental (Chaumier, 2004).

\section{Etapas de desarrollo}

La metodología empleada consta de tres etapas: La primera se denomina colecta y consiste en recopilar información, la segunda llamada tratamiento permite procesar dicha información y la tercera de nombre difusión establece la comunicación de los resultados.

Etapa 1. Colecta.- Se realizó una recopilación de información y se consideraron los trabajos de titulación desarrollados hasta el 2015 en la Carrera de Computación, se tomó la información relevante al proceso metodológico empleado para el desarrollo de dichos trabajo, obteniendo un total de 81 registros.

Etapa 2. Tratamiento.- Se estructuró una base de datos con información relevante al proceso metodológico de cada trabajo de titulación. Se analizaron los datos recabados, considerando que eran de variables cualitativas nominales, se realizó un estudio descriptivo y un estudio de series de casos para determinar las metodologías empleadas. De las líneas de investigación establecidas en la Carrera de Computación: Soluciones de hardware y aplicaciones de software; se procedió a categorizar los trabajos de titulación por sublínea de investigación a los trabajos propuestos bajo la línea de soluciones de hardware, ya que esta línea de investigación es de carácter muy amplio. Siguiente a esto, surgieron varias sublíneas, siendo una de ellas infraestructura tecnológica.

Se efectuó el análisis de las metodologías de esta sublínea, para determinar la frecuencia con la que se empleó, asignando un código para su identificación. Se procedió a determinar el nivel de pertinencia de cada metodología empleada en correspondencia con la investigación tecnológica desarrollada, esto se lo efectuó en base al análisis bibliográfico y su concordancia con el trabajo realizado.

Se procedió a realizar un análisis comparativo entre las fases de todas las metodologías empleadas, para determinar sus semejanzas y aplicabilidad, con este criterio, se dio paso a la propuesta de la metodología Eder, realizando la evaluación de la propuesta mediante el análisis de las fases de Eder con la información metodológica obtenida de los trabajos de titulación en la sublínea infraestructura tecnológica.

Etapa 3. Difusión.- De acuerdo a la metodología es de importancia difundir los resultados obtenidos de cada etapa, por ello se utilizaron estrategias como ponencias en eventos académicos-científicos para dar a conocer la propuesta metodológica ante la comunidad.

\section{Diseño de la propuesta}

Se determinó los trabajos de titulación en esta área (81) y se validó la metodología empleada, distribuida de acuerdo con las líneas de investigación de la carrera de Computación de la ESPAM MFL. Las líneas de investigación son: Soluciones de Hardware con 21(25.93\%) trabajos y Aplicaciones de Software con $60(74.07 \%)$. La línea Soluciones de Hardware, se categorizó por sublíneas de investigación, tal como se muestra en la tabla 1. 
Tabla 1: Trabajos de titulación de la línea Soluciones de Hardware, analizados por sublínea de investigación

\begin{tabular}{|l|c|c|}
\hline \multicolumn{1}{|c|}{ Sublíneas de Investigación } & $F$ & $\%$ \\
\hline Infraestructura Tecnológica & 10 & 47.64 \\
\hline Auditoría Informática & 4 & 19.05 \\
\hline Análisis de Riesgos & 2 & 9.52 \\
\hline Electrónica & 5 & 23.82 \\
\hline Total & 21 & 100.00 \\
\hline
\end{tabular}

De la clasificación realizada se puede observar que la sublínea Infraestructura Tecnológica cuenta con 10 trabajos de titulación, sobre los que se desarrolló un tratamiento analítico de los procesos metodológicos empleados. Al determinar la metodología y la frecuencia de su aplicación (Tabla 2), se aprecia la diversidad de metodologías empleadas, siendo en su gran mayoría para desarrollo de software o áreas de gestión de procesos, las que han sido adaptadas para fines de infraestructura tecnológica.

Tabla 2: Metodologías utilizadas en la sublínea de investigación Infraestructura Tecnológica

\begin{tabular}{|c|l|c|c|}
\hline Código & \multicolumn{1}{|c|}{ Metodología } & $F$ & $\%$ \\
\hline M1 & Diagrama Funcional GRAFCET & 1 & 10.00 \\
\hline M2 & Método de Hardware Libre & 2 & 20.00 \\
\hline M3 & Método Inductivo-deductivo & 1 & 10.00 \\
\hline M4 & Método SoftCal & 1 & 10.00 \\
\hline M5 & Metodología en V & 1 & 10.00 \\
\hline M6 & Modelo Cascada & 2 & 20.00 \\
\hline M7 & Modelo Cascada Modificado & 1 & 10.00 \\
\hline M8 & Modelo de Desarrollo ITIL & 1 & 10.00 \\
\hline & Total & 10 & 100.00 \\
\hline
\end{tabular}

Para determinar el criterio de pertinencia, se realizó una revisión de la metodología empleada, su campo de aplicación, así como la concordancia en el uso que se le dio dentro del trabajo efectuado. En la tabla 3 se detalla el criterio empleado.

Tabla 3: Criterios para determinar la pertinencia

\begin{tabular}{|l|l|l|}
\hline \multicolumn{1}{|c|}{$\begin{array}{c}\text { Campo de } \\
\text { aplicación }\end{array}$} & $\begin{array}{c}\text { Concordancia en } \\
\text { el trabajo }\end{array}$ & \multicolumn{1}{|c|}{$\begin{array}{c}\text { Criterio de } \\
\text { pertinencia }\end{array}$} \\
\hline \multirow{3}{*}{$\begin{array}{l}\text { Infraestructura } \\
\text { tecnológica }\end{array}$} & General & Total \\
\cline { 2 - 3 } & Parcial & Aceptable \\
\cline { 2 - 3 } Hardware & Escasa o ninguna & Regular \\
\hline \multirow{4}{*}{ Software } & General & Aceptable \\
\cline { 2 - 3 } & Parcial & Regular \\
\cline { 2 - 3 } & Escasa o ninguna & Escasa \\
\hline \multirow{3}{*}{$\begin{array}{l}\text { Gestión de } \\
\text { servicios de TI }\end{array}$} & General & Regular \\
\cline { 2 - 3 } & Parcial & Escasa \\
\cline { 2 - 3 } & Escasa o ninguna & Ninguna \\
\hline \multirow{4}{*}{ Otro } & General & Aceptable \\
\cline { 2 - 3 } & Earcial & Regular \\
\hline & Gena o ninguna & Escasa \\
\cline { 2 - 3 } & Parcial & Regular \\
\cline { 2 - 3 } & Escasa o ninguna & Escasa \\
\hline
\end{tabular}

La pertinencia que tiene cada una las metodologías empleadas se muestran en la tabla 4. Para este resultado se consideró el análisis bibliográfico de las metodologías utilizadas, su campo de aplicación y la concordancia al aplicarla en los trabajos de titulación de la sublínea infraestructura tecnológica. Se pudo apreciar que ninguno es totalmente pertinente y únicamente el $20 \%$ tiene una pertinencia aceptable (Figura 1). 
Tabla 4: Pertinencia metodológica según tema de infraestructura tecnológica: Pertinencia: total, aceptable, regular, escasa, ninguna

\begin{tabular}{|c|c|c|c|c|c|c|}
\hline No. & Cód. & Tema & Metodología & Campo & Concordancia & Pertinencia \\
\hline 1 & M1 & $\begin{array}{l}\text { Control de acceso del personal docente y } \\
\text { estudiantes en las aulas de la carrera } \\
\text { informática de la ESPAM-MFL } \\
\end{array}$ & $\begin{array}{l}\text { Diagrama } \\
\text { Funcional } \\
\text { GRAFCET } \\
\end{array}$ & Otros & Parcial & Escasa \\
\hline 2 & M2 & $\begin{array}{l}\text { Implementación de equipos de monitoreo y } \\
\text { seguridad basado en cámaras IP en el } \\
\text { almacén Lindón García Representaciones del } \\
\text { cantón Tosagua }\end{array}$ & $\begin{array}{l}\text { Método de } \\
\text { Hardware } \\
\text { Libre }\end{array}$ & Hardware & General & Aceptable \\
\hline 3 & M2 & $\begin{array}{l}\text { Implementación de un sistema de } \\
\text { comunicación con telefonía IP en el hotel } \\
\text { Higuerón de la ESPAM MFL }\end{array}$ & $\begin{array}{l}\text { Método de } \\
\text { Hardware } \\
\text { Libre }\end{array}$ & Hardware & General & Aceptable \\
\hline 4 & M3 & $\begin{array}{l}\text { Implementación de una intranet en la escuela } \\
\text { superior politécnica agropecuaria de Manabí } \\
\text { "Manuel Félix López" de la ciudad de Calceta } \\
\text { - cantón Bolívar }\end{array}$ & $\begin{array}{l}\text { Método } \\
\text { Inductivo- } \\
\text { deductivo }\end{array}$ & Otros & Parcial & Escasa \\
\hline 5 & M4 & $\begin{array}{l}\text { Vigilancia electrónica monitoreable } \\
\text { remotamente en las oficinas centrales de la } \\
\text { Escuela Superior Politécnica Agropecuaria de } \\
\text { Manabí Manuel Félix López }\end{array}$ & $\begin{array}{l}\text { Método } \\
\text { SoftCal }\end{array}$ & Software & $\begin{array}{l}\text { Escasa o } \\
\text { ninguna }\end{array}$ & Ninguna \\
\hline 6 & M5 & $\begin{array}{l}\text { Sistema de vigilancia mediante cámaras IP } \\
\text { en las oficinas de la Capitanía del Puerto de } \\
\text { la ciudad de Manta. }\end{array}$ & $\begin{array}{l}\text { Metodología } \\
\text { en V }\end{array}$ & Software & $\begin{array}{l}\text { Escasa o } \\
\text { ninguna }\end{array}$ & Ninguna \\
\hline 7 & M6 & $\begin{array}{l}\text { Telefonía IP entre los departamentos del Área } \\
\text { Agroindustrial de la Escuela Superior } \\
\text { Politécnica Agropecuaria de Manabí "Manuel } \\
\text { Félix López" }\end{array}$ & $\begin{array}{l}\text { Modelo } \\
\text { Cascada }\end{array}$ & Software & $\begin{array}{l}\text { Escasa o } \\
\text { ninguna }\end{array}$ & Ninguna \\
\hline 8 & M6 & $\begin{array}{l}\text { Vigilancia monitoreable por medio de } \\
\text { cámaras IP con motor de seguimiento en el } \\
\text { colegio Raymundo Aveiga de la ciudad de } \\
\text { Chone }\end{array}$ & $\begin{array}{l}\text { Modelo } \\
\text { Cascada }\end{array}$ & Software & $\begin{array}{l}\text { Escasa o } \\
\text { ninguna }\end{array}$ & Ninguna \\
\hline 9 & M7 & $\begin{array}{l}\text { Modelo alternativo; red de área local con } \\
\text { tecnología power line comunication en el } \\
\text { Colegio Nacional Técnico "Raymundo } \\
\text { Aveiga" de la ciudad de Chone } \\
\end{array}$ & $\begin{array}{l}\text { Modelo } \\
\text { Cascada } \\
\text { Modificado }\end{array}$ & Software & $\begin{array}{l}\text { Escasa o } \\
\text { ninguna }\end{array}$ & Ninguna \\
\hline 10 & M8 & $\begin{array}{l}\text { Implementación de una intranet en el Colegio } \\
\text { Fiscal Técnico Raymundo Aveiga de la } \\
\text { ciudad de Chone provincia de Manabí }\end{array}$ & $\begin{array}{l}\text { Modelo de } \\
\text { Desarrollo } \\
\text { ITIL } \\
\end{array}$ & $\begin{array}{l}\text { Gestión de } \\
\text { servicios de } \\
\text { TI }\end{array}$ & Parcial & Regular \\
\hline
\end{tabular}

Pertinencia

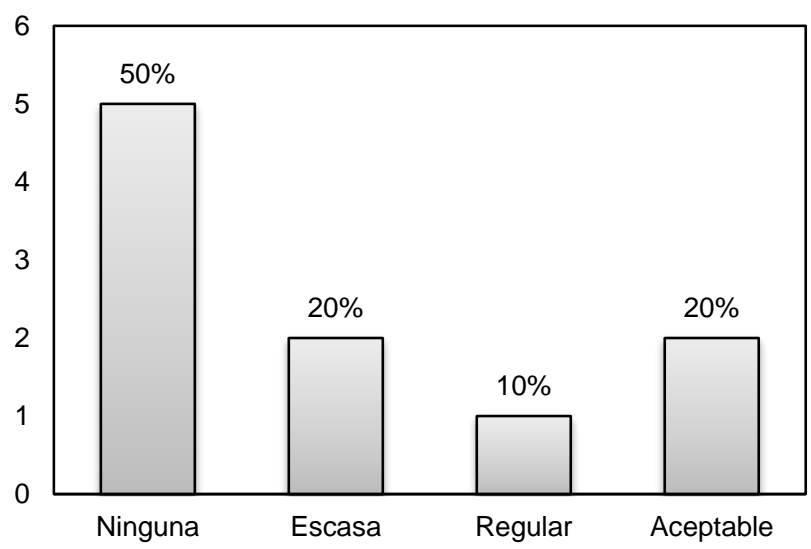

Fig. 1: Análisis Porcentual de la pertinencia de las metodologías

A partir del análisis de pertinencia, se realizó un estudio comparativo por las fases de cada metodología empleada en los trabajos de titulación para determinar semejanzas entre ellas. Se pudo establecer que muchas fases con nombres diferentes tienen similitudes conceptuales o de procedimientos, por tal razón se procedió a agruparlas de acuerdo a dichas similitudes, resultando 11 grupos (Tabla 5). 
Tabla 5: Análisis comparativo entre fases de las metodologías

\begin{tabular}{|c|c|c|c|c|c|c|c|c|c|c|}
\hline \multirow{2}{*}{ No. } & \multirow{2}{*}{ Fases del método } & \multicolumn{8}{|c|}{ Metodología } & \multirow{2}{*}{$F$} \\
\hline & & M1 & M2 & M3 & M4 & M5 & M6 & M7 & M8 & \\
\hline G1 & Observación/investigación preliminar & & & $\mathrm{X}$ & & $x$ & & & & 2 \\
\hline G2 & $\begin{array}{l}\text { Análisis de requisitos/análisis de } \\
\text { procesos/conceptualización }\end{array}$ & $X$ & $\mathrm{X}$ & & $\mathrm{X}$ & $\mathrm{X}$ & $X$ & $\mathrm{X}$ & $x$ & 7 \\
\hline G3 & Experimentación & & & $\mathrm{X}$ & & & & & & 1 \\
\hline G4 & $\begin{array}{l}\text { Diseño del sistema/diseño del } \\
\text { programa/arquitectura/administració } \\
\mathrm{n}\end{array}$ & & $\mathrm{X}$ & & $\mathrm{X}$ & $\mathrm{X}$ & $X$ & $\mathrm{X}$ & $\mathrm{X}$ & 6 \\
\hline G5 & Desarrollo/construcción/modelado & $x$ & $\mathrm{x}$ & & $x$ & $\mathrm{X}$ & $\mathrm{x}$ & $\mathrm{X}$ & $\mathrm{x}$ & 7 \\
\hline G6 & Comparación & & & $\mathrm{x}$ & & & & & & 1 \\
\hline G7 & Abstracción & & & $\mathrm{x}$ & & & & & & 1 \\
\hline G8 & Pruebas/verificación & $x$ & & & $x$ & $x$ & $x$ & $x$ & $x$ & 6 \\
\hline G9 & Implantación/evaluación/aceptación & & & & $X$ & $X$ & $X$ & $X$ & & 4 \\
\hline G10 & Generalización & & & $\mathrm{X}$ & & & & & & 1 \\
\hline G11 & Mantenimiento/cambios & & & & & & $\mathrm{X}$ & $\mathrm{X}$ & $\mathrm{X}$ & 3 \\
\hline
\end{tabular}

Una vez procesada la información, se determinó que existen 4 grupos de fases que son consideradas en la mayoría de las metodologías estudiadas, siendo estas G2 (Análisis de requisitos / Análisis de procesos / Conceptualización), G4 (Diseño del sistema / Diseño del programa / Arquitectura / Administración), G5 (Desarrollo / Construcción / Modelado), y G8 (Pruebas / Verificación).

\section{Propuesta}

De acuerdo con lo observado y analizado, los 4 grupos de etapas son las que actúan directamente en la ejecución técnica de todo trabajo de infraestructura tecnológica. Ante ello se plantea una metodología que contemple en sus fases los aspectos observados y pertinentes para proyectos de este tipo, incorporando además aspectos relevantes. La metodología propuesta se la ha identificado con el acrónimo Eder, por las siglas de las fases que la conforman: estudio, diseño, ejecución y revisión. En la figura 2 se plantea un esquema gráfico de la propuesta metodológica.

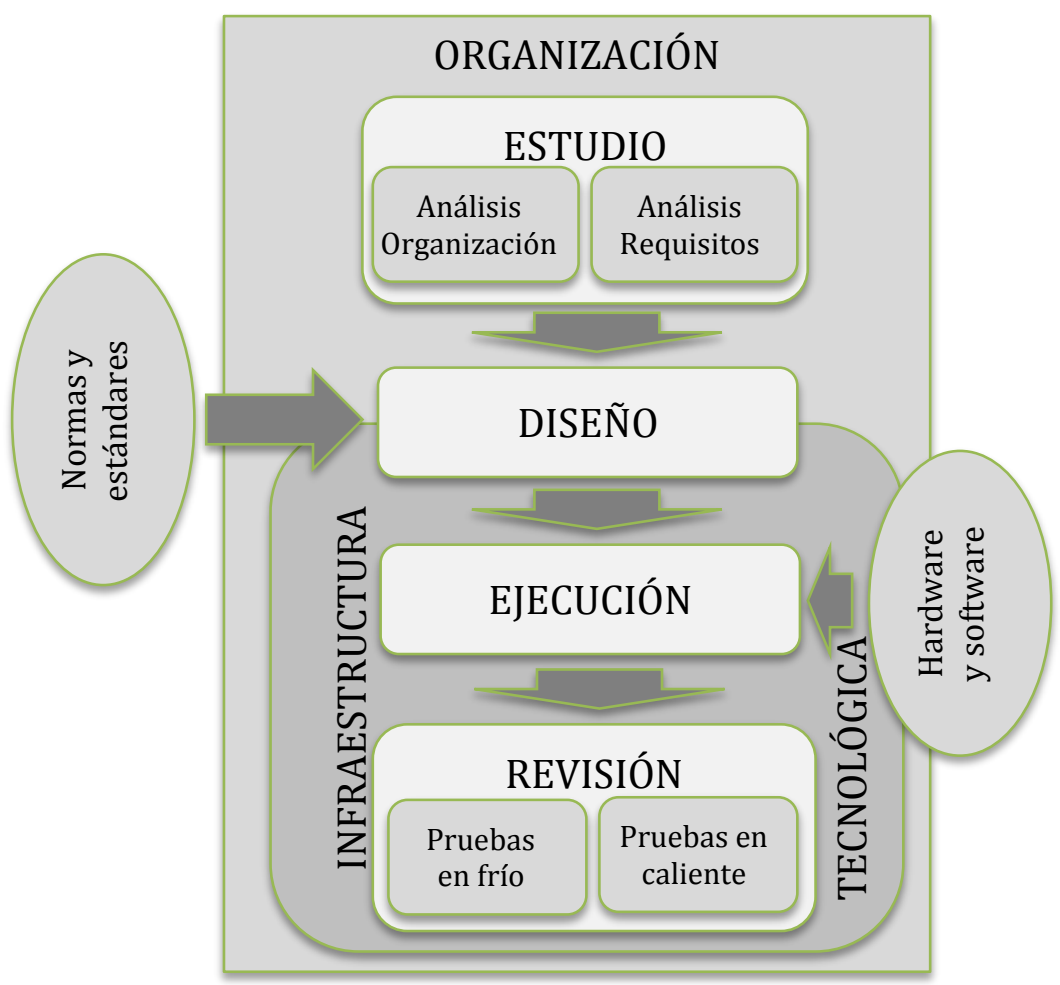

Fig. 2: Metodología Eder 
Estudio.- Una infraestructura tecnológica tiene como objetivo satisfacer las necesidades de negocio de una organización, de tal forma que los procesos se vuelvan más eficientes, facilitando las comunicaciones y el intercambio de información. En esta etapa se plantearon dos actividades:

Análisis de la organización: El trabajo comienza analizando todos los componentes de la organización, así como los requisitos que les permitan alcanzar los objetivos organizacionales, ya que la infraestructura es siempre parte de un sistema mayor,

Análisis de los requisitos: Se centra e intensifica especialmente los aspectos de servicio de comunicación, soporte a la información, servicios de procesamiento de datos, entre otros.

Diseño.- Traduce los requisitos en una representación técnica de la infraestructura a implantarse, considerando la calidad (normas, estándares, entre otros) requerida antes que comience la ejecución. Se debe estipular una arquitectura que sea robusta pero flexible, de tal forma que permita cambios en el futuro. Se detallarán claramente las características de los componentes de hardware y software que se integrarán. Será necesario determinar en esta etapa un cronograma de trabajo que contemple desde la ejecución hasta el proceso de pruebas respectivas.

Ejecución.- Se desarrolla partiendo del diseño de la solución. Se debe implementar con base en la arquitectura proyectada en la fase anterior, integrando los diferentes componentes de hardware y software, y siguiendo estándares de calidad de acuerdo a los componentes y actividades planificadas.

Revisión.- Aquí también se comprende dos actividades, cuya finalidad es verificar el correcto funcionamiento de la solución de infraestructura tecnológica desarrollada, tanto en ambiente no productivo, como en producción.

Pruebas en frío: Son verificaciones de acuerdo con diferentes tipos de pruebas planificadas para comprobar la integración de cada uno de los diferentes componentes como parte de la solución propuesta.

Pruebas en caliente: Son verificaciones basadas en métricas que se planifican para verificar el correcto funcionamiento de la solución en ambiente de producción. Se establece de antemano un periodo de tiempo prudencial que permitirá corregir errores (de darse) y finalizar con la aceptación a satisfacción del cliente.

En cada una de las fases y actividades será indispensable hacer uso de instrumentos que faciliten documentar el cumplimiento de cada una de ellas.

\section{Validación}

Para la validación de la metodología se diseñó una matriz (Tabla 6) donde se valoró los diez proyectos de la sublínea de infraestructura tecnológica y sus métodos empleados frente a la metodología Eder. De manera resumida se ubicó el contenido del desarrollo metodológico de cada trabajo de titulación en correspondencia con cada fase de la metodología propuesta. Se prosiguió, con el desarrollo de una rúbrica, considerando el aspecto de adaptabilidad del proceso metodológico, con varios niveles de equivalencia, siendo estos: Total $\rightarrow 100 \%$, Aceptable $\rightarrow 75 \%$, Regular $\rightarrow 50 \%$, Escasa $\rightarrow 25 \%$ y Ninguna $\rightarrow 0 \%$.

Tabla 6: Equivalencia de las metodologías investigadas vs metodología EDER. CM=Cascada modificado; $H L=$ Hardware libre; C=Cascada; I= Inductivo; $\mathrm{S}=$ Softcal

\begin{tabular}{|l|c|c|c|c|c|c|c|c|c|c|c|}
\hline Trabajo de Titulación & TT1 & TT2 & TT3 & TT4 & TT5 & TT6 & TT7 & TT8 & TT9 & TT10 & \multirow{2}{*}{ Total } \\
\hline Metodología & $I$ & CM & C & $S$ & Itil & HL & Grafcet & En v & C & HL & \\
\hline Estudio & $75 \%$ & $75 \%$ & $100 \%$ & $100 \%$ & $100 \%$ & $25 \%$ & $25 \%$ & $50 \%$ & $75 \%$ & $50 \%$ & $68 \%$ \\
\hline Diseño & $0 \%$ & $50 \%$ & $100 \%$ & $100 \%$ & $50 \%$ & $25 \%$ & $75 \%$ & $100 \%$ & $100 \%$ & $25 \%$ & $63 \%$ \\
\hline Ejecución & $100 \%$ & $100 \%$ & $100 \%$ & $100 \%$ & $100 \%$ & $50 \%$ & $50 \%$ & $100 \%$ & $100 \%$ & $75 \%$ & $88 \%$ \\
\hline Revisión & $50 \%$ & $75 \%$ & $50 \%$ & $75 \%$ & $75 \%$ & $50 \%$ & $50 \%$ & $50 \%$ & $25 \%$ & $25 \%$ & $53 \%$ \\
\hline Total & $56 \%$ & $75 \%$ & $88 \%$ & $94 \%$ & $81 \%$ & $38 \%$ & $50 \%$ & $75 \%$ & $75 \%$ & $44 \%$ & $68 \%$ \\
\hline
\end{tabular}

En la tabla 6 se visualiza que el trabajo de titulación 4 (TT4), tuvo mayor adaptabilidad metodológica en un $94 \%$ en correspondencia con Eder, ya que posee una cantidad de información suficiente para poder proceder con la aplicación propuesta en esta investigación. Es importante resaltar que, de acuerdo con la información proporcionada e interpretación realizada, la metodología cascada a pesar de ser utilizada en TT3 y TT9, difieren los resultados en cuanto a la adaptabilidad, lo que deja entrever que, a pesar de hacer uso de la 
misma metodología, no siempre se aplica de igual manera su guía metodológica, esto hace que varíen los resultados. Lo mismo ocurre en el caso de la aplicación de la metodología Hardware libre, empleada en TT6 y TT10.

Se puede identificar de manera clara que la propuesta de la metodología Eder, proporciona procesos claros y pertinentes en la aplicación de proyectos de infraestructura tecnológica, siendo en las fases Estudio y Revisión más exigentes, para la culminación exitosa de la propuesta. El alto porcentaje obtenido en la etapa de ejecución muestra que la mayoría de las propuestas enfocan o centran su realización en el desarrollo del trabajo, Eder como alternativa metodológica, determina la importancia en establecer de manera clara el estudio de la organización, antes del diseño y ejecución de la solución. Además, la verificación tanto en frío como en producción, de acuerdo con los autores, propende al aseguramiento de un mayor nivel de éxito en proyectos de infraestructura tecnológica.

Se debe considerar que los sistemas de ciencia, tecnología e innovación son extremadamente complejos en cualquier país del mundo, tal como lo expresa Díaz (2009), así mismo manifiesta que esto se debe a ser, en su mayoría, autónomos y por consiguiente heterogéneos. Estas razones pueden explicar la dificultad para representar en forma homóloga sus procesos en los diferentes países. Díaz recalca también, que en América Latina este escenario es aún más complejo e incide incluso en la visibilidad de sus resultados. Eso es consecuencia de la desarticulación entre las metodologías para la medición de estos aspectos a escala internacional y los indicadores propios de los contextos latinoamericanos.

Werlinger et al. (2014) manifiesta que las instituciones universitarias tienen como parte de su misión la generación de nuevo conocimiento a partir del ejercicio científico de su comunidad académica y estudiantil, proceso fundamental en toda disciplina al permitir su desarrollo, orientar la toma de decisiones y diseñar planes y programas en el mediano y largo plazo.

Se pretende entonces, que la metodología propuesta, sea base para el desarrollo metodológico de investigaciones orientadas a infraestructura tecnológica en diferentes contextos, sean estos nacionales o internacionales, que con sus fases sencillas y de fácil comprensión aseguren el desarrollo exitoso de toda propuesta de titulación en el área en mención., teniendo en cuenta que las metodologías deben considerar el trabajo en equipo y las habilidades de revisión crítica (Silva et al., 2017).

\section{CONCLUSIONES}

Esta investigación ha permitido demostrar que las metodologías utilizadas para el desarrollo de trabajos de titulación son base fundamental para asegurar resultados óptimos y alcanzar los objetivos propuestos, también deja una visión clara de que los procesos metodológicos empleados no siempre son utilizados adecuadamente y con pertinencia.

Las etapas de las diversas metodologías utilizadas, aún en la diversidad de nombres que poseen, convergen en cuanto a su conceptualización, lo que permitió agruparlas y determinar aquellas con mayor frecuencia en las diferentes metodologías utilizadas en trabajos de titulación de infraestructura tecnológica, permitiendo de esta manera estructurar las etapas de la propuesta Eder.

Se verificó un nivel aceptable de concordancia en la aplicabilidad (68\%) de otras metodologías empleadas frente a Eder, es necesario considerar que se comparó a los diez trabajos de titulación de la sublínea de infraestructura tecnológica, esto también permitió demostrar que los trabajos de titulación, en su gran mayoría, omiten información relevante en el proceso del desarrollo de la ejecución técnica, lo que conlleva a trabajos poco reproducibles o replicables.

La propuesta de la metodología Eder, establece procesos sencillos, claros y pertinentes, estableciendo de manera concreta las etapas con su flujo de trabajo, esta alternativa metodológica, determina la importancia en establecer en su etapa inicial el estudio de la organización, antes del diseño y ejecución de la solución. La etapa de revisión tanto en frío como en producción asegura un mayor nivel de éxito para la culminación en proyectos de infraestructura tecnológica.

\section{REFERENCIAS}

Agostinho, O. L., Proposal of Organization Framework Model, using Business Processes and Hierarchical Patterns to Provide Agility and Flexibility in Competitiveness Environments, Procedia Engineering, 131, 401-409 (2015)

Aguilera, R., Identidad y Diferenciación entre Método y Metodología, Estudios Políticos, 9(28), 81-103 (2013)

Carrillo, G., A. Azamar y G. Torre-Marin, Innovación Tecnológica y Curtiduría en el Estado de Guanajuato, Economía Informa, 402, 66-79 (2017) 
Chaumier, J., Les techniques documentaires, 9e édition, Presses Universitaires de France, Paris (2004)

Da Silva, D., R. Vieira, A. Vieira y M. De Santiago, Optimización del Proceso de Innovación para Proyectos Internos en las Empresas, doi: 10.4067/S0718-07642016000300011, Información Tecnológica, 27(3), 119-130 (2016)

De Oliveira, R., L. García, A. Sandoval, R. De Sousa y K. Tai-Hoon, Leveraging Information Security and Computational Trust for Cybersecurity, doi: 10.1007/s11227-015-1543-4, J. Supercomput., 72, 3729-3763 (2016)

Díaz, M., Situación de las Metodologías para la Medición de la Ciencia, la Tecnología y la Innovación en América Latina, Acimed, 19(4), 1-7 (2009)

Ley-Bosch, C., I. Alonso-González, D. Sánchez-Rodríguez y C. Ramírez-Casañas, Evaluation of the Effects of Hidden Node Problems in IEEE 802.15.7 Uplink Performance, doi: 10.3390/s16020216, Sensors, 16, 216 (2016)

Mair, C., Taking Technological Infrastructure Seriously: Standards, Intellectual Property and Open Access, doi: 10.5334/ujiel.266, Utrecht Journal of International and European Law, 32(82), 59-88 (2016)

Martelo, R., I. Blanquicet y L. Rodríguez, Metodología para Seleccionar Aplicaciones de la Tecnología de Comunicaciones Voz sobre la IP (VoIP) para Pequeñas y Medianas Empresas (Pymes). El caso de una Agencia de Viajes, doi: 10.4067/S0718-07642015000600014, Información Tecnológica, 26(6), 121-128 (2015)

Martelo, R., I. Jiménez-Pitre y L. Moncaris, Guía Metodológica para el Mejoramiento del Desarrollo de Software a través de la Aplicación de la Técnica Árboles de Problemas, doi: 10.4067/S0718-07642017000300010, Información Tecnológica, 28(3), 87-94 (2017)

Montesinos-Valera, J., P. Aragonés-Beltrán y J. Pastor, Selection of Maintenance, Renewal and Improvement Projects in Rail Lines Using the Analytic Network Process, doi: 10.1080/15732479.2017.1294189, Structure and Infrastructure Engineering, 13, 11, 1476-1496 (2017)

Navia, M., J. Campelo, A. Bonastre y R. Ors, GTSO: Global Trace Synchronization and Ordering Mechanism for Wireless Sensor Network Monitoring Platforms, doi: 10.3390/s16020216, Sensors, 18(1), 28 (2018)

Pereira, J. y J. Oliveira, Process-Cased Information Systems: Technological Infrastructure and Development Issues, Procedia Computer Science, 100, 872-877 (2016)

Petty, N., O. Thomson y G. Stew, Ready for a Paradigm shift? Part 2: Introducing Qualitative Research Methodologies and Methods, Manual Therapy, 17, 378-384 (2012)

Plan Nacional de Desarrollo 2017-2021, Senplades, Ecuador (2017)

Plinio, P., S. Oviedo y D. Franco, Metodología para el Diagnóstico de Prácticas del Modelo Proceso Personal de Software, doi: 10.4067/S0718-07642014000200007, Información Tecnológica, 25(2), 57-66 (2014)

Porcal-Gonzalo, M., A Strategy for the Management, Preservation, and Reutilization of Geographical Information Based on the Lifecycle of Geospatial Data: An Assessment and a Proposal Based on Experiences from Spain and Europe, doi: 10.1080/15420353.2015.1064054, Journal of Map \& Geography Libraries, 11, 3, 289-329 (2015)

Scheel, C. y D. Bretones, The Impact of Technology-based Clusters on Regional Development: The Case of the Grand Poitiers Futuroscope Technopole, Revista Universidad \& Empresa, 20, 11-43 (2011)

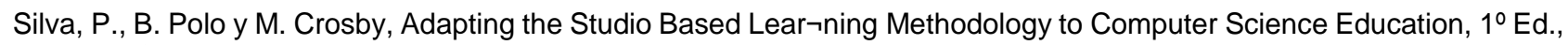
119-142, Springer International Publishing (2017)

Torres-Velandia, S., D. Ruíz-Ávila y L. Meza-López, Infraestructura y Equipamiento Tecnológico en los Doctorados Consolidados de Conacyt: una Mirada desde las Políticas Públicas y los Académicos, Universia RIES Revista Iberoamericana de Educación Superior, 21, VIII (2017)

Werlinger, F., L. Coronado, C. Ulloa, O. Candia y G. Rojas, Metodología y Visibilidad en los Medios de Divulgación Científica de las Tesis de Pregrado en Odontología. Educación Médica Superior, 28(2), 318-334 (2014) 
